\title{
On the maximum mass of hyperonic neutron stars
}

\author{
É. Massot ${ }^{1,2}$, J. Margueron ${ }^{1}$, and G. Chanfray ${ }^{3}$ \\ 1 Institut de Physique Nucléaire, IN2P3-CNRS and Université Paris-Sud, F-91406 Orsay CEDEX, France \\ 2 Département de physique, École Normale Supérieure, 24 rue Lhomond, 75231 Paris Cedex 05, France, \\ 3 IPN Lyon, Université de Lyon, Univ. Lyon 1, CNRS/IN2P3, UMR5822, F-69622 Villeurbanne Cedex
}

PACS 97.60.Jd-

PACS 26.60.Kp-

\begin{abstract}
Chiral Lagrangian and quark-meson coupling models of hyperon matter are used to estimate the maximum mass of neutron stars. Our relativistic calculations include, for the first time, both Hartree and Fock contributions in a consistent manner. Being related to the underlying quark structure of baryons, these models are considered to be good candidates for describing the dense core of neutron stars. Taking account of the known experimental constraints at saturation density, the equations of state deduced from these relativistic approaches cannot sustain a neutron star with a mass larger than 1.6-1.66 $M_{\odot}$.
\end{abstract}

Neutron stars are the most compact stellar objects, and within them, particles that are unstable on earth could be stabilized at densities above $10^{14} \mathrm{~g} \mathrm{~cm}^{-3}$. The equation of state (EoS) at these densities is still largely unknown due $\checkmark$ to the poor experimental knowledge on hadron dynamics. However, the recent measurements of the millisecond pulSars PSR J1614-2230 extend the maximum observed mass from $(1.67 \pm 0.02) \mathrm{M}_{\odot}$ [1] to $(1.97 \pm 0.04) \mathrm{M}_{\odot}$ [2]. This imposes new constrains on the EoS of dense matter [3]. A major question is whether the EoS including hyperons can be ruled out by this new observation. Relativistic meanfield [4,5] and Brueckner-Hartree-Fock [6, 8] models have predicted that the effect of hyperons in dense matter is to soften the EoS and, therefore, to lower the maximum mass. A consistent treatment of the Fock term in a relativistic approach is however missing. In this Letter we analyze the impact of the Fock term in two effective, relativistic meson-exchange models related to the quark nature of hadrons: the chiral Lagrangian model [9] and the quark meson coupling (QMC) model [10].

These models are based on a relativistic meson-exchange theory of the Walecka type, where the mean-field properties of nuclear matter are calculated in the Hartree-Fock approximation [11. Considering scalar $s, \omega, \delta, \rho$ and $\pi$ mesons as well as nucleons and hyperons, the Lagrangian can be written with the usual notations 10

$$
\mathcal{L}=\sum_{f} \bar{\psi}_{f}\left(i \gamma^{\mu} \partial_{\mu}-M_{f}(s)\right) \psi_{f}-V(s)+\frac{1}{2} \partial^{\mu} s \partial_{\mu} s
$$

$$
\begin{aligned}
& -\sum_{f} \bar{\psi}_{f} g_{\omega}^{f} \omega^{\mu} \gamma_{\mu} \psi_{f}+\frac{1}{2} m_{\omega}^{2} \omega_{\mu} \omega^{\mu}-\frac{1}{4} F^{\mu \nu} F_{\mu \nu} \\
& -\quad g_{\delta} \sum_{f} \bar{\psi}_{f} \vec{\delta} \cdot \vec{I} \psi_{f}-\frac{1}{2} m_{\delta}^{2} \vec{\delta} \cdot \vec{\delta}+\frac{1}{2} \partial^{\mu} \vec{\delta} \cdot \partial_{\mu} \vec{\delta} \\
& -\quad g_{\rho} \vec{\rho}^{\mu} \cdot \sum_{f} \bar{\psi}_{f} \gamma_{\mu} \vec{I} \psi_{f}-g_{\rho} \frac{\kappa_{\rho}}{2 M_{N}} \partial_{\nu} \vec{\rho}_{\mu} \cdot \sum_{f} \bar{\psi}_{f} \bar{\sigma}^{\mu \nu} \vec{I} \psi_{f} \\
& +\frac{1}{2} m_{\rho}^{2} \vec{\rho}_{\mu} \cdot \vec{\rho}^{\mu}-\frac{1}{4} \vec{F}^{\mu \nu} \cdot \vec{F}_{\mu \nu}^{\prime}+\frac{g_{A}}{2 f_{\pi}} \frac{3}{5} \partial_{\mu} \vec{\pi} \cdot \sum_{f} \bar{\psi}_{f} \vec{G}_{T}^{\mu} \psi_{f} \\
& +\frac{1}{2} m_{\pi}^{2} \vec{\pi} \cdot \vec{\pi}-\frac{1}{2} \partial^{\mu} \vec{\pi} \cdot \partial_{\mu} \vec{\pi},
\end{aligned}
$$

where the sum over the flavor index $f$ means a summation over the baryons: N, $\Lambda, \Sigma, \Xi$. The isospin operator $\vec{I}$ and the Gamow-Teller operator $\vec{G}_{T}^{\mu}$ are given in Ref. [10].

In the chiral Lagrangian model [9], the meson fields are related to the properties of the QCD condensate. Scalar $s$ and pseudo-scalar $\pi$ fields are associated with the fluctuations of the chiral quark condensate [12, related to the non-linear "Mexican hat" potential,

$$
V(s)=\frac{m_{s}^{2}}{2} s^{2}+\frac{m_{s}^{2}-m_{\pi}^{2}}{2 f_{\pi}} s^{3}+\frac{m_{s}^{2}-m_{\pi}^{2}}{8 f_{\pi}^{2}} s^{4},
$$

deduced from chiral spontaneous symmetry breaking. In this model, the chiral partner $A_{1}$ of the $\rho$ meson, being too heavy, is ignored and the $\omega$ meson is chiral singlet. The first chiral models failed to reproduce the saturation properties of nuclear matter [12. A better description 
Table 1: Parameters of chiral and QMC models and saturation properties: density $\left(n_{0}\right)$, binding energy $\left(B_{0}\right)$, incompressibility $\left(K_{0}\right)$, symmetry energy $\left(a_{s}\right)$, symmetry energy slope $\left(L_{0}\right)$ and in-medium Dirac mass for nucleons $\left(M_{N}^{*}\right)$. QMC700 is given in Ref. [10.

\begin{tabular}{|c|c|c|c|c|c|c|c|c|c|c|c|c|}
\hline Model & $m_{s}$ & $g_{s}$ & $g_{\omega}$ & $g_{\rho}$ & $\begin{array}{c}\kappa_{\rho} \\
\left(\mathrm{fm}^{-3}\right)\end{array}$ & $\begin{array}{c}C \\
(\mathrm{MeV})\end{array}$ & $\begin{array}{c}n_{0} \\
(\mathrm{MeV})\end{array}$ & $\begin{array}{c}B_{0} \\
(\mathrm{MeV})\end{array}$ & $\begin{array}{c}K_{0} \\
(\mathrm{MeV})\end{array}$ & $\begin{array}{c}a_{s} \\
(\mathrm{MeV})\end{array}$ & $\begin{array}{c}L_{0} \\
(\mathrm{MeV})\end{array}$ & $M_{N}^{*} / M_{N}$ \\
\hline MC1-H & 800 & 10.0 & 5.06 & 4.14 & 0.0 & 1.44 & 0.160 & -15.85 & 248 & 30.0 & 79.4 & 0.89 \\
\hline MC1-HF & 880 & 10.0 & 7.09 & 2.65 & 4.2 & 2.00 & 0.160 & -16.01 & 274 & 30.3 & 66.9 & 0.81 \\
\hline MC2-HF & 860 & 10.0 & 7.31 & 2.65 & 4.2 & 1.90 & 0.160 & -16.05 & 276 & 30.6 & 67.9 & 0.80 \\
\hline MC3-HF & 900 & 10.0 & 6.33 & 2.65 & 5.6 & 1.92 & 0.160 & -15.91 & 274 & 30.2 & 63.1 & 0.83 \\
\hline MC4-HF & 880 & 10.0 & 6.57 & 2.65 & 5.6 & 1.83 & 0.160 & -15.92 & 274 & 30.5 & 64.1 & 0.82 \\
\hline QMC700 & 700 & 11.94 & 10.66 & 4.167 & 0.0 & 0.51 & 0.159 & -15.74 & 338 & 29.9 & 53.3 & 0.74 \\
\hline QMC-H & 700 & 10.52 & 8.39 & 4.167 & 0.0 & 0.40 & 0.160 & -15.83 & 292 & 32.7 & 89.5 & 0.78 \\
\hline QMC-HF1 & 700 & 11.90 & 10.62 & 4.167 & 0.0 & 0.51 & 0.160 & -15.72 & 338 & 30.4 & 54.4 & 0.77 \\
\hline QMC-HF2 & 700 & 11.30 & 9.31 & 2.87 & 0.0 & 0.46 & 0.160 & -15.74 & 360 & 29.8 & 72.2 & 0.67 \\
\hline
\end{tabular}

for the saturation is obtained by introducing the scalar polarizability of the nucleon $\kappa_{N S}$ in $M_{f}(s)$ [13, 14,

$$
M_{f}(s)=M_{f}+g_{s} w_{s}^{f} s+\frac{1}{2} \kappa_{N S} \tilde{w}_{s}^{f} s^{2},
$$

which reflects the effects of confinement as originally proposed in Ref. [15. The weight factors $w_{s}^{f}$ and $\tilde{w}_{s}^{f}$ will be discussed later.

A further extension of the model consists in replacing $\kappa_{N S}$ in Eq. (3) by $\kappa_{N S}(s)=\kappa_{N S}\left(1+(2 s) /\left(3 f_{\pi}\right)\right)$ where $f_{\pi}$ is the decay constant of the pion [9. Another extension is related to the $\rho$ and $\pi$ tensor interactions, where derivative couplings lead to a contact term that can be partially or totally suppressed by short-range correlations [9,11. We consider chiral models at the Hartree approximation (such as MC1-H in table 1) as well as in the Hartree-Fock approximation (such as MCi-HF in table 1). The index $i$ in $\mathrm{MC} i-\mathrm{H}(\mathrm{F})$ models refers to different combinaisons of the extensions discussed: $(i=1)$, constant $\kappa_{N S}$ in Eq. (3) and totally suppressed contact term; $(i=2) \kappa_{N S}(s)$ in Eq. (3) and totally suppressed contact term; $(i=3)$ constant $\kappa_{N S}$ and partially suppressed contact term; $(i=4) \kappa_{N S}(s)$ and partially suppressed contact term. More details on these extensions can be found in Ref. [9].

In the quark-meson coupling model QMC700 the scalar potential (2) is quadratic, $V(s)=m_{s}^{2} s^{2} / 2$, at variance with the chiral Lagrangian model (2); the scalar polarizability of the nucleon $\kappa_{N S}$ is kept constant as in Eq. (3); and only $s, \omega$ and $\rho$ vector mesons are considered $\left(\kappa_{\rho}=0\right)$. The other extensions presented here-before are disregarded. In Ref. 10, self-energies are calculated in the Hartree approximation while a non-relativistic approximation of the Fock contribution to the energy is included. In this paper, we present modified versions of the QMC model in which both Hartree and Fock contributions are treated consistently.

The parameters of chiral Lagrangian and QMC models are given in table 1 In the chiral model, the scalar coupling constant is fixed from the sigma linear model to be $g_{s}=M_{N} / f_{\pi}=10$. Since the dimensionless parameter $C=f_{\pi}^{2} \kappa_{N S} /\left(2 M_{N}\right)$ estimated from lattice simulation is around $1.25[18$, the chiral model fixes $C$ to be consistent with this value but a flexibility is retained for reproducing saturation properties as explained below. In the QMC model, the dimensionless parameter $C$ is smaller and deduced from the nucleon mass in the bag model. The coupling constant $g_{\rho}$ is adjusted to fit the symmetry energy in the chiral Hartree model MC1-H and in QMC models while in chiral Hartree-Fock models $\mathrm{MC} i$-HF $g_{\rho}$ is fixed to satisfy the vector-dominance model giving $g_{\rho}=2.65$ and the $\rho$-tensor coupling constant $\kappa_{\rho}$ is adjusted to the symmetry energy. The axial coupling constant is fixed to be $g_{A}=1.25$ and the $\delta$ coupling is fixed to $g_{\delta}=1$. The other parameters $\left(m_{s}, g_{\omega}\right.$ and $\left.C\right)$ are chosen to reproduce the saturation properties $\left(n_{0}, B_{0}\right.$ and $\left.K_{0}\right)$ reported on table 1. In QMC model, the couplings $g_{s}$ and $g_{\omega}$ are fixed to reproduce $n_{0}$ and $B_{0}$; moreover in the parameterization QMC-HF3 the parameter $C$ is modified to decrease the value of $K_{0}$.

The predictions for the slope of the symmetry energy $L_{0}$ and the nucleon Dirac mass $M_{N}^{*}$ given in table $\square$ for the two models are consistent with experimental values lying in the range $60-90 \mathrm{MeV}$ [16] for the slope and in the range $0.7-0.9 M_{N}$ for the Dirac masses.

Hyperons are included as in Ref. [10: the coupling constants are written as $g_{m}^{f}=w_{m}^{f} g_{m}$ where $w_{m}^{f}$ is the weight factor for the meson $m$ coupled to the baryon $f$. For the mesons $\omega, \rho, \pi$ and $\delta$, the meson-hyperon couplings are deduced from the meson-nucleon couplings based on $S U(6)$ symmetry imposing $w_{\omega}^{f}=\left(1+s^{f} / 3\right)$, where $s^{f}$ 
Table 2: Weight factor $w_{s}^{f}$ for the hyperons $\Lambda, \Sigma$ and $\Xi$.

\begin{tabular}{lccc}
\hline \hline Model & $\Lambda$ & $\Sigma$ & $\Xi$ \\
\hline MC1-H & 0.61 & 0.18 & 0.33 \\
MC1-HF & 1.00 & 0.50 & 0.55 \\
MC2-HF & 1.00 & 0.51 & 0.52 \\
MC3-HF & 0.91 & 0.38 & 0.48 \\
MC4-HF & 0.89 & 0.39 & 0.47 \\
\hline QMC-H & 0.62 & 0.35 & 0.32 \\
QMC-HF1 & 0.71 & 0.51 & 0.37 \\
QMC-HF2 & 0.64 & 0.41 & 0.33 \\
QMC-HF3 & 0.70 & 0.33 & 0.37 \\
\hline \hline
\end{tabular}

is the strangeness of the baryon $f$. For the isovector $\rho$ or pseudo-vector $\pi$ mesons, this symmetry is accounted for by the isospin and Gamow-Teller operators. Only the coupling constants of the scalar $s$ meson are allowed to vary around the values imposed by $S U(6)$ symmetry. In QMC700, the weights $w_{s}^{f}$ and $\tilde{w}_{s}^{f}$ are adjusted in the bag model and scale with the bag radius [10. In the chiral model and in the extensions of QMC in table 1, they are adjusted to the non-relativistic potentials of hyperons at saturation,

$$
V_{N R}(f)=\Sigma_{S}^{f}+\frac{E^{f}}{M^{f}(s)} \Sigma_{0}^{f}+\frac{\Sigma_{S}^{f 2}-\Sigma_{0}^{f 2}}{2 M^{f}(s)},
$$

where $E^{f}$ is the single-particle energy and $\Sigma_{S}^{f}$ and $\Sigma_{0}^{f}$ are the scalar and time-component self-energies [9]. $V_{N R}(f)$ is fixed to be $-30 \mathrm{MeV}$ for $f=\Lambda$ [17, $30 \mathrm{MeV}$ for $f=\Sigma$ [19] and $-18 \mathrm{MeV}$ for $f=\Xi$ [4. While the value for the $\Lambda$ is quite certain, that for the other hyperons are still under debate. The weights $w_{s}^{f}$ are given in table 2 while we choose $\tilde{w}_{s}^{f}=\left(1+s^{f} / 3\right)$ for all baryons $f$.

Being partly related to the underlying quark structure of baryons the chiral and QMC models are good candidates for exploration of the properties of dense core of neutron stars. In table 3 we explore different models for the EoS with and without hyperons and their predictions for the maximum mass $M_{\max }$ and associated radius $R\left(M_{\max }\right)$. The first three EoS are based only on nucleons. For the following three models in table 3. the EoS is based on non-interacting hyperons. The maximum mass for these models is strongly reduced compared to models without hyperons. Interacting hyperons are taken into account in the next 10 models in table 3. For the five chiral models the maximum mass is increased compared to the models with free hyperons, but it remains smaller than that predicted by the models without hyperons. There is a small variation of about $0.05 M_{\odot}$ between the different $\mathrm{HF}$ models depending on the extension of the chiral model under consideration.

In the last four lines of table 3 we present variants of
Table 3: Different models corresponding to different approximations for nucleons (second column) and for hyperons (third column). The last three columns correspond to the maximum mass $M_{\max }$, its associated radius $R_{\max }$ and the corresponding baryonic density $n_{\max }^{c}$.

\begin{tabular}{|c|c|c|c|c|c|}
\hline EoS & $\begin{array}{c}\text { Nucleon } \\
\mathrm{N} \\
\end{array}$ & $\begin{array}{c}\text { Hyperon } \\
\text { Y }\end{array}$ & $\begin{array}{l}M_{\max } \\
\left(M_{\odot}\right) \\
\end{array}$ & $\begin{array}{c}R\left(M_{\max }\right) \\
(\mathrm{km})\end{array}$ & $\begin{array}{c}n_{\max }^{c} \\
\left(\text { in } n_{0}\right) \\
\end{array}$ \\
\hline $\mathrm{MC} 1-\mathrm{H} / \mathrm{N}$ & MC1-H & No & 1.65 & 10.6 & 7.8 \\
\hline MC1-HF/N & MC1-HF & No & 1.87 & 10.8 & 7.2 \\
\hline MC3-HF/N & MC3-HF & No & 1.85 & 10.7 & 7.3 \\
\hline $\mathrm{MC} 1-\mathrm{H} / \mathrm{NY}_{F G}$ & MC1-H & Fermi Gas & 1.02 & 11.9 & 5.6 \\
\hline $\mathrm{MC1}-\mathrm{HF} / \mathrm{NY}_{F G}$ & MC1-HF & Fermi Gas & 1.12 & 12.0 & 5.4 \\
\hline $\mathrm{MC} 3-\mathrm{HF} / \mathrm{NY}_{F G}$ & MC3-HF & Fermi Gas & 1.13 & 11.9 & 5.5 \\
\hline $\mathrm{MC} 1-\mathrm{H} / \mathrm{NY}$ & MC1-H & MC1-H & 1.45 & 11.9 & 6.5 \\
\hline MC1-HF/NY & MC1-HF & MC1-HF & 1.58 & 12.2 & 5.6 \\
\hline $\mathrm{MC} 2-\mathrm{HF} / \mathrm{NY}$ & $\mathrm{MC} 2-\mathrm{HF}$ & $\mathrm{MC} 2-\mathrm{HF}$ & 1.60 & 12.2 & 5.5 \\
\hline MC3-HF/NY & MC3-HF & MC3-HF & 1.55 & 12.0 & 5.6 \\
\hline MC4-HF/NY & MC4-HF & MC4-HF & 1.55 & 12.0 & 5.6 \\
\hline QMC700/NY & QMC700 & QMC700 & 2.00 & 12.5 & 5.1 \\
\hline QMC-H/NY & QMC-H & QMC-H & 1.68 & 12.2 & 5.5 \\
\hline QMC-HF1/NY & QMC-HF1 & QMC-HF1 & 1.97 & 12.3 & 5.2 \\
\hline QMC-HF2/NY & QMC-HF2 & QMC-HF2 & 1.97 & 13.3 & 4.6 \\
\hline QMC-HF3/NY & QMC-HF3 & QMC-HF3 & 1.66 & 12.3 & 5.3 \\
\hline
\end{tabular}

the QMC model whose parameters are given in table 1 . The original QMC700/NY model predicts a maximum mass of $2.00 \mathrm{M}_{\odot}$ with hyperons [10]. The model QMC$\mathrm{H} / \mathrm{NY}$ is a consistent Hartree model where the $s$ and $\omega$ coupling constants are readjusted to reproduce saturation properties $\left(n_{0}, B_{0}\right.$ and $\left.V_{N R}(f)\right)$. The maximum mass in the model QMC-H/NY is lower than that of the original QMC700/NY since the incompressibility is lower. In the model QMC-HF1/NY the Hartree and Fock terms are consistently treated in the mean-field potential and the same readjustment procedure as in QMC-H/NY is followed. The Fock contribution to the total energy is however treated non-relativistically as in the QMC700/NY model. Including the complete Fock contribution in the energy, we obtain the fully relativistic QMC-HF2/NY model. The maximum mass is not altered by the fully relativistic treatment of the Fock term. Finally, we reduced the incompressibility by increasing the parameter $C$ from 0.51 to 1 (see table 1) and the maximum mass decreases from 1.97 to $1.66 \mathrm{M}_{\odot}$. From this analysis, we conclude that the large mass obtained in the QMC700/NY model [10] is mainly due to an overestimated and incompressibility.

We show in figure 1 the mass-radius relation for the various EoS presented in this Letter. Dotted lines show results for the models without hyperons while the solid lines stand for the models including interacting hyperons. 


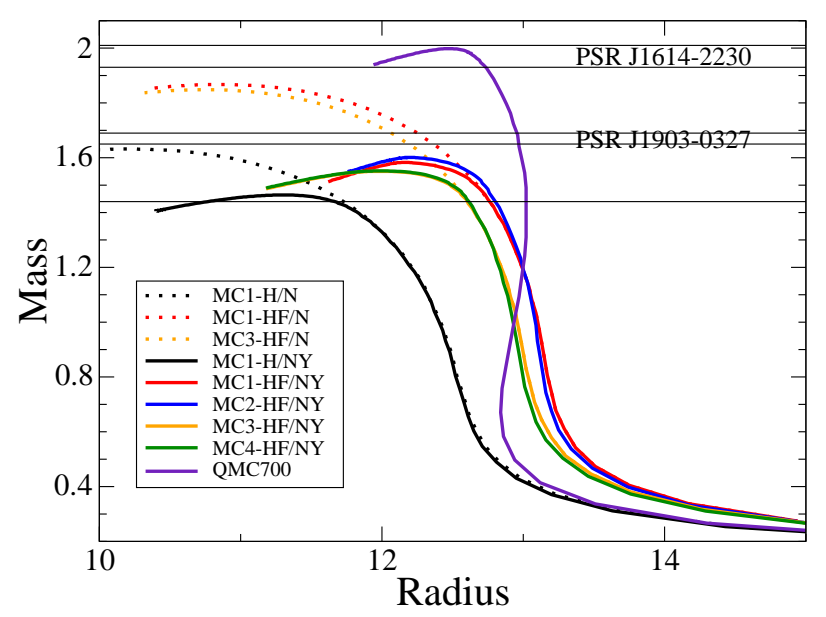

Fig. 1: Mass of neutron stars versus the radius for selected EoS.

As expected the EoS with hyperons reach lower maximum masses than the models without hyperons, except the model QMC700 due to its large and unrealistic incompressibility. The maximum mass obtained with hypernuclear EoS does not change much for different models. This is due to a self-regulating compensation effect between the softening of the EoS and the onset of the hyperonic degree of freedom [78]. The maximum mass of about $1.6 M_{\odot}$ predicted by the relativistic approach is slightly larger than that of $1.4 M_{\odot}$ found in Ref. [8]. The difference of $0.2 M_{\odot}$ is mainly related to the larger incompressibility of the relativistic models. It is also observed that the radii for the Hartree models are smaller than that of the Hartree-Fock ones, and that the predicted radii of the relativistic models and that of Ref. [8] are comparable.

The abundances for nucleons and hyperons are shown for various EoS in figure 2 with free hyperons (MC1$\mathrm{H} / \mathrm{NY}_{F G}$ ) and with interacting hyperons (MC1-H/NY and MC1-HF/NY). The order of appearance of hyperons is different for these models and depends strongly on the corresponding interactions. In the model $\mathrm{MC} 1-\mathrm{H} / \mathrm{NY}_{F}$ with free hyperons, the $\Sigma^{-}$is non interacting and appears first. On the other hand, in the models MC1-H/NY and MC1-HF/NY, the $\Sigma^{-}$interaction is repulsive while the $\Xi^{-}$ interaction is attractive, therefore the latter tends to prevent the $\Sigma^{-}$from appearing. In the model $\mathrm{MC} 1-\mathrm{H} / \mathrm{NY}$, the $\Lambda$ appears first followed closely in density by the $\Xi^{-}$, then at a larger density by the $\Xi^{0}$, while for the model MC1-HF/NY the $\Xi^{-}$and $\Xi^{0}$ appear first and push the other hyperons to larger densities.

We have presented in this Letter different EoS for hyperon matter derived from both chiral and QMC models. These models are thermodynamically consistent and treat the Hartree and Fock terms on equal footing. We have found that the different chiral parameterizations give a maximum neutron star mass that does not exceed $1.6 \mathrm{M}_{\odot}$ for $\mathrm{HF}$ models with $K_{0} \approx 270 \mathrm{MeV}$. Correcting the QMC700 model [10] by including the complete relativistic
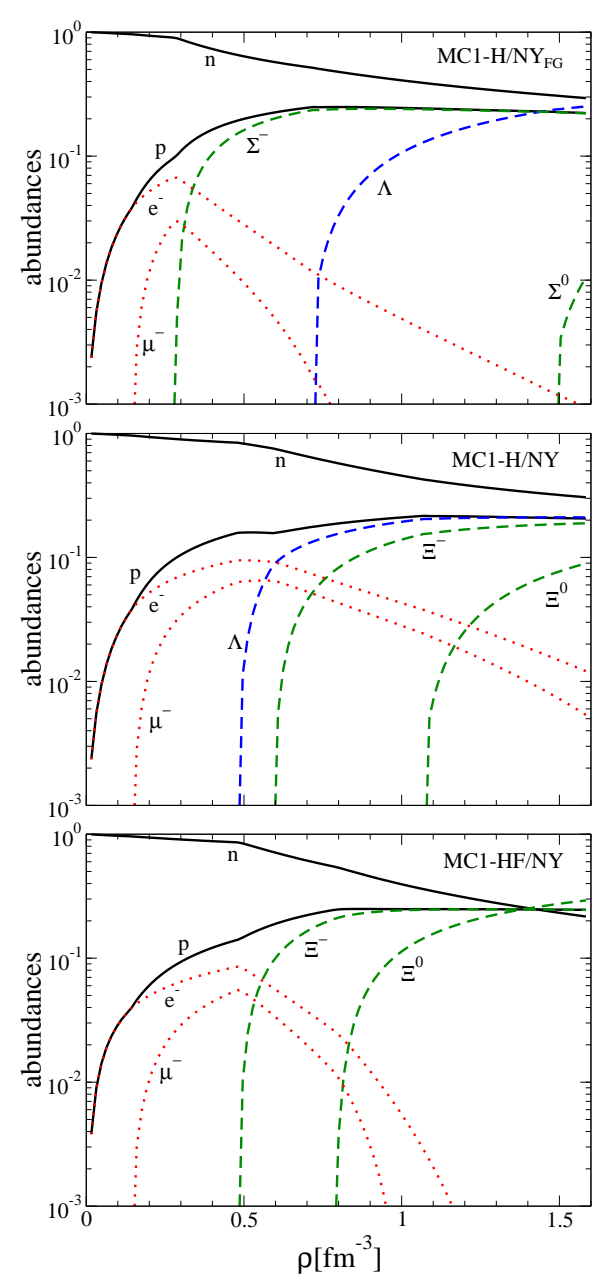

Fig. 2: Particules abundances for the various models of table 3 $\mathrm{MC} 1-\mathrm{H} / \mathrm{NY}_{F G}$ (top), MC1-H/NY (middle) and MC1-HF/NY (bottom).

contribution to the Fock term, a correct incompressibility and the experimental constraints for the hyperon meanfields, we found a decrease of the predicted maximum mass from 2.00 down to $1.66 \mathrm{M}_{\odot}$. We additionally checked that the effect of rotation with the period of $3.15 \mathrm{~ms}$ (as for PSR J1614-2230) does not increase the maximum mass by more than $0.02 M_{\odot}$. We therefore conclude that it is difficult to reconcile any of the model presented in this work with the observed mass of PSR J1614-2230 and the empirical knowledge of saturation properties of nuclear matter. Unless an unexpected property of the hyperon interaction is missing in the present model, as well as in the BHF models of Refs. 6, 8, 20, our results tends to exclude hypernuclear matter to be present in the core of massive neutron stars. It reinforces alternative models such as for instance the model of deconfined quark matter, for which experimental constraints are almost inexistent. We can therefore conclude that the equation of state of dense matter in the core of neutron star is still not even qualitatively understood. 
We thank I. Vidaña for fruitful discussions during the completion of this work. This work was partially supported by the ANR NExEN and SN2NS project ANR-10BLAN-0503 contracts and COMPSTAR, an ESF Research Networking Program.

\section{REFERENCES}

[1] S. M. Ransom, J. W. T. Hessels, I. H. Stairs, P. C. C. Freire, F. Camilo, V. M. Kaspi and D. L. Kaplan, Sience 307, 892 (2005).

[2] P. Demorest, T. Pennucci, S. M. Ransom, M. Roberts, J. W. T. Hessels, Nature 467, 1081 (2010).

[3] J. M. Lattimer and M. Prakash, To appear in Gerry Brown's Festscrift, World Scientific (2010), arXiv:1012.3208(astro-ph).

[4] J. Schafner-Bielich, M. Hanauske, H. Stöcker, and W. Greiner, Phys. Rev. Lett. 89, 171101 (2002).

[5] C. Wu, and Z. Ren, Phys. Rev. C 83, 025805 (2011).

[6] M. Baldo, G. F. Burgio, and H.-J. Schulze, Phys. Rev. C 61, 055801 (2000).

[7] H.-J. Schulze, A. Polls, A. Ramos, and I. Vidaña, Phys. Rev. 73, 058801 (2006).

[8] H.-J. Schulze and T. Rijken, Phys. Rev. 84, 035801 (2011).

[9] E. Massot, and G. Chanfray, Phys. Rev. C 78, 015204 (2008).

[10] J. Rikovska-Stone, P. A. M. Guichon, H. H. Matevosyan, A. W. Thomas, Nucl. Phys. A 792, 341 (2007).

[11] A. Bouyssy, J. F. Mathiot, N. Van Giai, and S. Marcos, Phys. Rev. C 36, 380 (1987)

[12] G. Chanfray, M. Ericson, P. A. M. Guichon, Phys. Rev. C 63, 055202 (2001)

[13] A. K. Kerman and L. D. Miller, In Berkeley 1974, Proceedings, Lawrence Berkeley Lab Lbl- 3675, Berkely, 73-107 (1974).

[14] W. Bentz, and A. W. Thomas, Nucl. Phys. A 696, 138 (2001).

[15] P. A. M. Guichon, Phys. Lett. B 200, 235 (1988).

[16] I. Vidaña, C. Providência, A. Polls, and A. Rios, Phys. Rev. C 80, 045806 (2009).

[17] D. J. Millener, C. B. Dover, and A. Gal, Phys. Rev. C 38, 2700 (1988).

[18] A. W. Thomas, P. A. M. Guichon, D. B. Leinweber, R. D. Young, Prog. Theor. Phys. Suppl. 156, 124 (2004).

[19] P. K. Saha, et al., Phys. Rev. C 70, 044613 (2004)

[20] I. Vidaña, D. Logoteta, C. Providência, A. Polls, I. Bombaci, Eur. Phys. Lett. 94, 11002 (2011). 